\title{
Revascularización en incisivo permanente joven no vital post traumatismo dental: Reporte de caso
}

\author{
Paola Rivas Escobar, ${ }^{1}$ \\ Gilmer Torres Ramos, ${ }^{2}$ \\ Roxana Patricia López Ramos, ${ }^{3}$
}

\section{Resumen}

El tratamiento de endodoncia en dientes permanentes jóvenes que han sufrido un episodio de trauma dental era desalentador hasta hace unas épocas. La revascularización, se ha posicionado como una opción de tratamiento que tienen aquellos dientes con raíces inmaduras porque favorece el desarrollo radicular y el posterior cierre apical. El objetivo fue describir el tratamiento de revascularización en un incisivo central permanente no vital post traumatismo dental. Se presenta el caso de un paciente de sexo femenino, de 08 años de edad, con antecedentes de traumatismo dental, en sector antero superior, incisivo central permanente con formación radicular incompleta y con ausencia de vitalidad pulpar; se realizó el tratamiento de revascularización pulpar utilizando pasta antibiótica 3Mix - MP para la desinfección del conducto radicular. Se realizaron controles clínicos y radiográficos para evaluar la ausencia de sintomatología y el desarrollo radicular, a los 14 meses se observó aumento del grosor y longitud radicular y cierre apical. Se concluyó que la revascularización es una opción de tratamiento que permite un desarrollo radicular en sentido longitudinal y un engrosamiento de las paredes del conducto radicular con un posterior cierre apical, disminuyendo el riesgo de fractura que pudiera sufrir.

Palabras clave: Traumatismo de los dientes, diente permanente, pulpa dental, antibiótico.

Especialista en Odontopediatría, Universidad Nacional Mayor de San Marcos.

2 PhD en Estomatología, MSc. en Gerencia en Servicios de Salud Especialista en Odontopediatría. Universidad Nacional Mayor de San Marcos.

${ }^{3} \mathrm{PhD}(\mathrm{c})$ en Ciencias en Investigación Epidemiológica. MSc en Estomatología, Especialista en Odontopediatría. Universidad Peruana Cayetano Heredia. 
Caso clinico

\section{Revascularização em incisivo permanente jovem não vital após traumatismo dentário: relato de caso}

\begin{abstract}
Resumo
O tratamento endodôntico de dentes permanentes jovens que sofreram um episódio de trauma dentário foi desencorajador até algumas vezes atrás. A revascularização foi posicionada como uma opção de tratamento para os dentes com raízes imaturas, pois favorece $\mathrm{o}$ desenvolvimento radicular e o fechamento apical subsequente. $\mathrm{O}$ objetivo foi descrever o tratamento de revascularização em um incisivo central permanente não vital após traumatismo dentário. Apresentamos o caso de uma paciente do sexo feminino, 8 anos, com história de traumatismo dentário, no setor antero superior, incisivo central permanente com formação radicular incompleta e ausência
\end{abstract}

de vitalidade pulpar; $\mathrm{O}$ tratamento de revascularização da polpa foi realizado com pasta antibiótica 3Mix-MP para desinfecção do canal radicular. Controles clínicos e radiográficos foram realizados para avaliar ausência de sintomas e o desenvolvimento radicular, aos 14 meses foi observado um aumento na espessura e comprimento da raiz e no fechamento apical. Concluiu-se que a revascularização é uma opção de tratamento que permite o desenvolvimento radicular na direção longitudinal e o espessamento das paredes do canal radicular com posterior fechamento apical, reduzindo o risco de fratura que poderia ser sofrida.

Palavra-chave: Traumatismos dentário, dente permanente, polpa Dentária, antibiótico.

Case report

\section{Revascularization in non-vital young permanent incisor post dental trauma: Case report}

\begin{abstract}
Endodontic treatment of immature permanent teeth that have had an episode of dental trauma was discouraging until a recently. Revascularization has been positioned as a treatment option because it promotes root development and subsequent apical closure. The aim is of this report is to describe revascularization treatment performed in a non-vital permanent central incisor with history of dental trauma. We present the case
\end{abstract}

of an 8 year-old female patient of with history of dental trauma, in the upper anterior section, The permanent central incisor presented with incomplete root formation and absence of pulpal vitality. Pulp revascularization treatment was performed using 3Mix - MP paste for disinfection of the root canal. Clinical and radiographic controls were performed to evaluate the absence of symptomatology and root development. After 14 months the thickness and root length and the apical closure were increased. It was 
concluded that the revascularization is a treatment option that allows root development in the longitudinal direction and a thickening of the walls

\section{Introducción}

Los dientes inmaduros con pulpas no vitales, son consideradas un reto en el tratamiento endodóntico; siendo la mayor complicación un foramen apical de gran tamaño y falta de desarrollo radicular. ${ }^{1,2,3}$

El tratamiento tradicional para este tipo de dientes era la apexificación, el cual se basa en la formación de un tabique apical a modo de ápice y teniendo como desventaja paredes radiculares delgadas, divergentes y propensas a la fractura. ${ }^{4}$ Actualmente, se plantea realizar el tratamiento de revascularización pulpar en este tipo de casos, el cual favorecerá el desarrollo radicular basándose en la desinfección del conducto radicular, ${ }^{1-3,5-10}$ de este modo se proporciona una matriz de tejido estéril en el que nuevas células pueden crecer y la vitalidad de la pulpa puede ser restablecida. ${ }^{11}$

El tratamiento de revascularización consta de dos citas, la segunda cita se puede realizar a $\operatorname{los} 7,14$ o 21 días, y sólo si el diente se encuentra asintomático y se evidencia una reducción de la lesión apical. $^{3,6,9}$ El éxito del tratamiento de revascularización pulpar va a depender de tres elementos: desinfección del canal radicular, presencia de andamio (coágulo de sangre) con células mesenquimales y sellado hermético coronario. ${ }^{1,12-14}$ Para ello, debemos tener en cuenta las siguientes consideraciones en el procedimiento de revascularización pulpar: of the root canal with an apical posterior closure, decreasing the risk of fracture.

Keywords: Tooth Injuries, permanent tooth, Dental Pulp, antibiotic.

La mayoría de estudios recomienda una instrumentación mínima (ampliación del $1 / 3$ coronal del conducto radicular $)^{15}$ o nula ${ }^{6,13}$ Esto debido a lo delgado de las paredes dentinales y hacerlas más proclives a las fracturas. ${ }^{13,15}$

La irrigación puede ser con Hipoclorito de Sodio $(\mathrm{NaClO})$ pues tiene una acción disolvente sobre el tejido necrótico y es un potente agente antimicrobiano ${ }^{6,7}$ y se recomienda su uso en una concentración de 2,5\% para evitar la toxicidad en tejidos adyacentes. ${ }^{6}$ Otra alternativa es la Clorhexidina ( $\mathrm{CHX}$ ), en una concentración de $2 \%$, tiene propiedades antimicrobianas y baja citotoxicidad ${ }^{6}$ y según estudios, es segura si este medicamento entra en contacto con los tejidos periapicales en dientes inmaduros., ${ }^{75-17}$ También recomiendan es el uso de $\mathrm{NaClO}$ y $\mathrm{CHX}$ $2 \%$ como irrigantes, siendo el irrigante inicial el $\mathrm{NaClO}$ seguido de la irrigación de solución salina (cloruro de Sodio) para prevenir posible interacción entre $\mathrm{NaClO}$ y $\mathrm{CHX}$ que es utilizado como irrigante final $2,5,15,18,19$, de este modo evitar la formación de un precipitado (paracloroanilina -PCA), que es un conocido carcinógeno. ${ }^{2,5,15}$

La Pasta 3 Mix es usada comúnmente en la desinfección de los conductos radiculares, por la capacidad que tiene de difundir a través estos hasta la zona periapical y consta de una parte líquida (macrogol y propelinglicol) y una de polvo (minociclina, metronidazol 
y ciprofloxacino). ${ }^{20-22}$ El tiempo que permanece la pasta $3 \mathrm{Mix}$ en el conducto radicular es de dos semanas. ${ }^{13,20,21}$ Estudios demuestran, que la combinación de la instrumentación químico-mecánica, asociada a una terapia medicamentosa con pasta 3 Mix reduce los niveles bacterianos hasta un $70 \% .^{13,20}$

El coágulo (fibrina reticulada) actúa como un andamio el cual es esencial para ayudar al crecimiento del nuevo tejido en el espacio del canal. 5, 7,9,10 La inducción del sangrado en los conductos radiculares se puede realizar al irritar los tejidos apicales con un explorador de endodoncia o una lima estéril, ${ }^{15}$ el cual debe realizar un sobrepase de $2 \mathrm{~mm}$ del extremo apical, 2,5 permitiendo que el conducto radicular se llene de sangre $3 \mathrm{~mm}$ por debajo del límite amelocementario. ${ }^{2,7}$ Se debe evitar el uso de anestésicos locales con epinefrina para facilitar el sangrado. ${ }^{15,23}$ Tiempo promedio para la formación de un coágulo estable es de 15 minutos. ${ }^{6,23}$

La barrera cervical-sellado se realiza con MTA, este se coloca sobre el coágulo estable formado en la zona cervical. ${ }^{5,8}$ Aproximadamente, $3 \mathrm{~mm}$ de MTA es colocado en el tercio coronal (unión cemento-esmalte) ${ }^{21}$ haciendo uso de un porta amalgama ${ }^{4}$, éste tiene un tiempo de fraguado de más de 2,5 horas y alcanza un sellado ideal a las 48 horas8. Algunos estudios recomiendan realizar la restauración final 2 días después de culminada la segunda cita de revascularización. ${ }^{24}$

El acceso coronal debe ser adecuadamente sellado para prevenir la reinfección. ${ }^{13}$ La decoloración de la corona clínica ocurre por el uso de minociclina como componente de la pasta $3 \mathrm{Mix}$ o el uso de
MTA. ${ }^{15,25}$ Algunos estudios recomiendan la sustitución de minociclina por cefalexina para evitar dicha decoloración coronal. ${ }^{16,20}$

Los controles deben realizarse cada 3 meses durante el primer año y luego cada 6 meses hasta que se observe el cierre apical., ${ }^{2,5}$ Se apreciará resolución de lesión periapical a los 6 meses, y elongación radicular y cierre apical con engrosamiento de las paredes del conducto radicular en un período de 12 a 24 meses. ${ }^{2-4}$

Las ventajas de este procedimiento son: la regeneración del tejido en el conducto radicular con células sanguíneas propias del paciente, acortar el tiempo de tratamiento, reduce la fragilidad radicular, se puede evidenciar radiográficamente el desarrollo radicular. ${ }^{26} \mathrm{Y}$ las desventajas son: falta de seguimiento a largo plazo, cambio de coloración a nivel coronal, resistencia bacteriana, reacción alérgica por el uso de medicamentos y no existe un protocolo universal. ${ }^{23}$

\section{Reporte de caso}

Se presenta el caso de un paciente de sexo femenino, de 8 años de edad. La paciente acudió acompañada con su padre al servicio de endodoncia del Instituto Nacional de Salud del Niño en LimaPerú, con antecedentes de traumatismo dental, en sector antero superior y con dolor espontáneo. $\mathrm{Al}$ examen clínico intraoral, se evaluó que el diente 11 presentaba una ligera movilidad dental y al examen radiográfico se observó diente 11 no vital, ligero ensanchamiento del ligamento periodontal y desarrollo radicular en estadio 8 de Nolla (Figura 
1 y 2). Se evaluó el caso y se realizó la explicación de las ventajas y desventajas del tratamiento de revascularización pulpar al padre de familia y se procedió a la firma del consentimiento informado para el tratamiento.

La secuencia de tratamiento se realizó en 2 sesiones: la primera sesión se basó en la desinfección del conducto radicular, utilizando como agente irrigante inicial $20 \mathrm{ml}$ de hipoclorito de sodio al 2,5\% y $10 \mathrm{ml}$ clorhexidina al $2 \%$, como irrigante final, intercalando la irrigación con $10 \mathrm{ml}$ de cloruro de sodio, para evitar la formación de precipitados; ;,5,15,18,19 luego el conducto radicular fue secado con

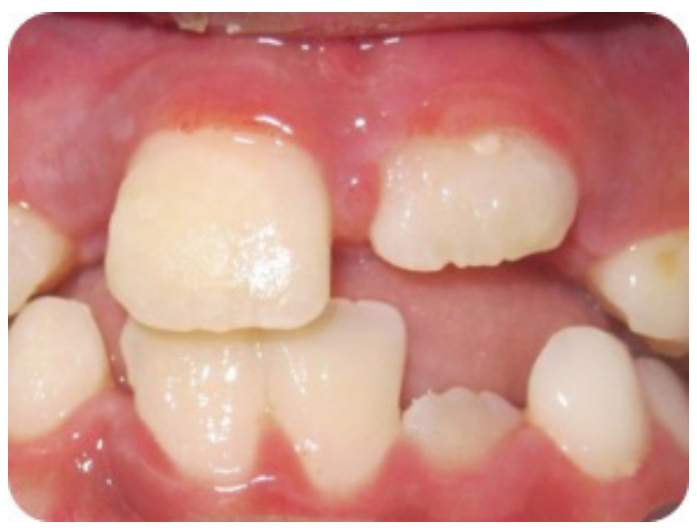

Figura 1. Pza. 11 con antecedentes de traumatismo alveolo dentario.

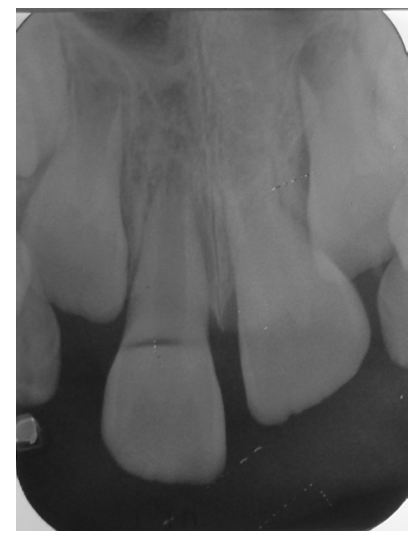

Figura 2. Radiografía periapical de diagnóstico. conos de papel estériles. Finalmente, en el conducto radicular se colocó la pasta antibiótica 3Mix (Figura 3-5), mezclado previamente según las indicaciones de Quintana y cols. ${ }^{22}$ La segunda sesión, se realizó a las 2 semanas, ${ }^{22}$ lográndose la inducción del sangrado (formación del

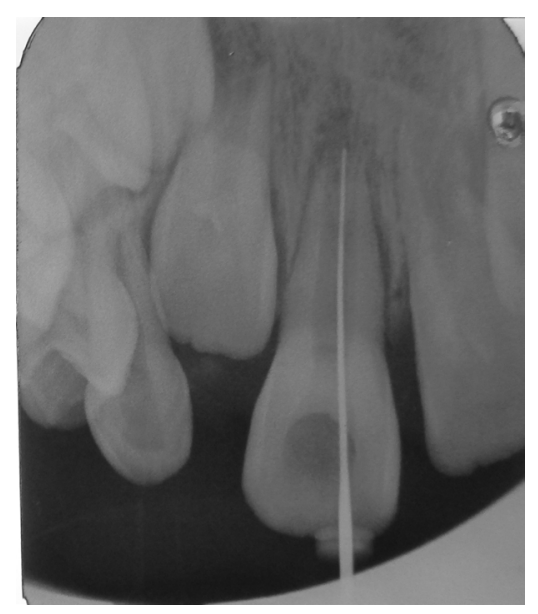

Figura 3. Apertura cameral con piedra diamantada redonda a nivel de la cara palatina. Conductometría con lima $K N^{\circ} 35$ en una longitud de $17 \mathrm{~mm}$. Control radiográfico.

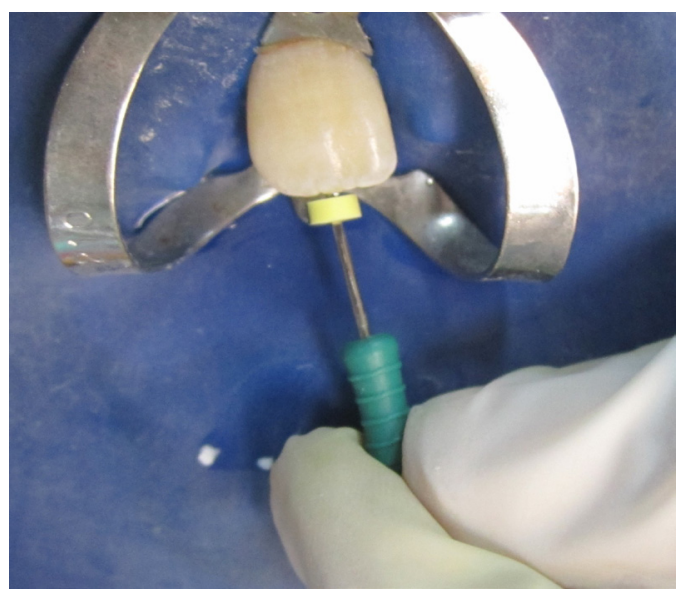

Figura 4. Preparación biomecánica con lima K $N^{\circ} 35$, en una longitud de $12 \mathrm{~mm}$. Sólo a nivel cervical para mejorar visibilidad y el acceso al conducto radicular. Irrigación inicial con hipoclorito de sodio al $2.5 \%$ y $10 \mathrm{ml}$ clorhexidina al $2 \%$, como irrigante final, intercalando la irrigación con $10 \mathrm{ml}$ de cloruro de sodio. 


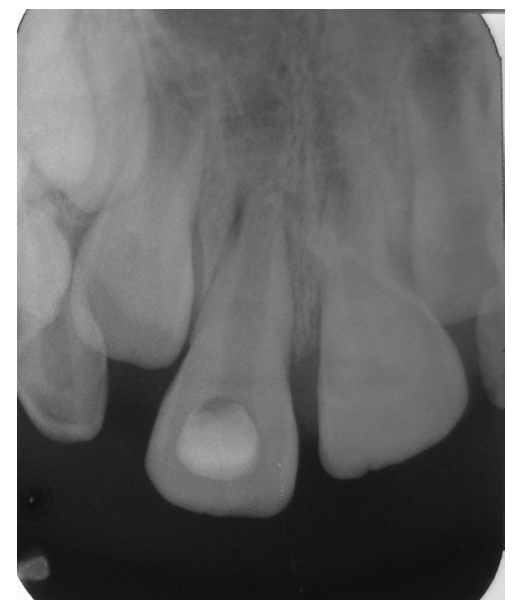

Figura 5. Aplicación de Pasta 3 Mix intra conducto con lima $\mathrm{K}$ № 25 . El sellado de la entrada del conducto radicular se realizó con pasta de eugenato + ionómero de vidrio por un perído de 15 días. Control radiográfico.

andamio) y colocándose una barrera cervical de MTA. El éxito del tratamiento de revascularización pulpar también depende en gran parte de una adecuada reconstrucción y sellado a nivel coronal, en este caso se realizó utilizando materiales ionoméricos y resinosos (Figura 6-10).

Después de realizada la revascularización y la colocación de la restauración

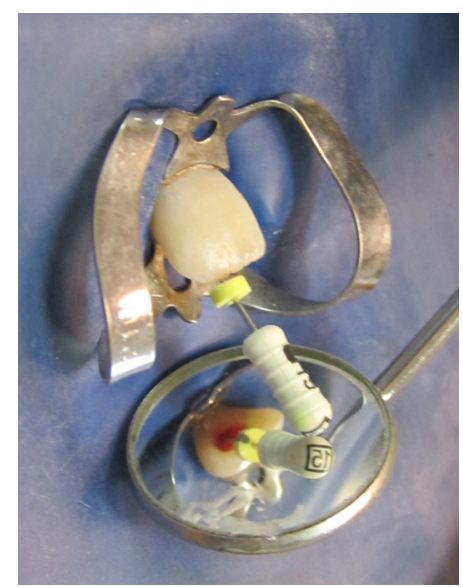

Figura 6. Remoción de pasta de 3 Mix. Estimulación de sangrado con lima $K N^{\circ} 15$ aumentando de 1- $2 \mathrm{~mm}$ de la conductometría inicial.

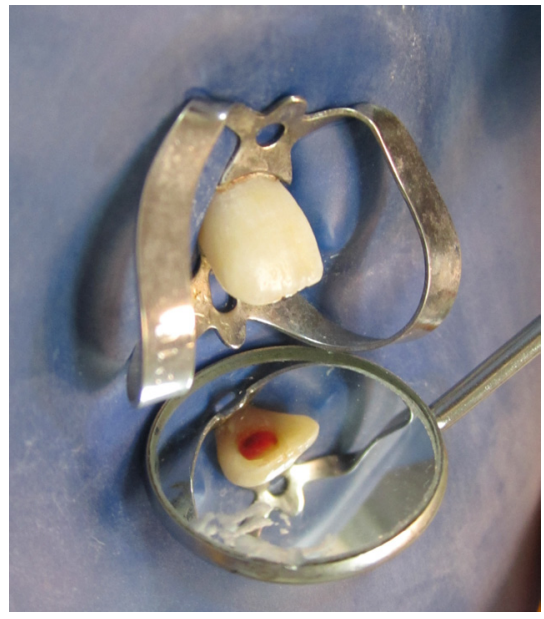

Figura 7. Se dejó que se estabilice el coágulo por 15 minutos.

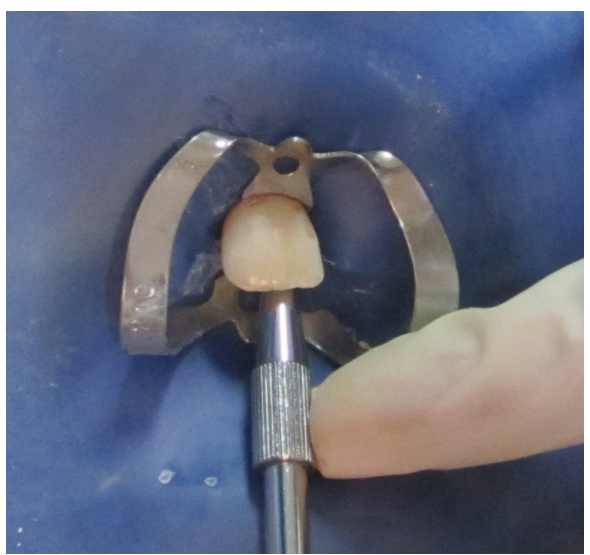

Figura 8. Se aplicó MTA con una porta amalgama sobre el coágulo estable.

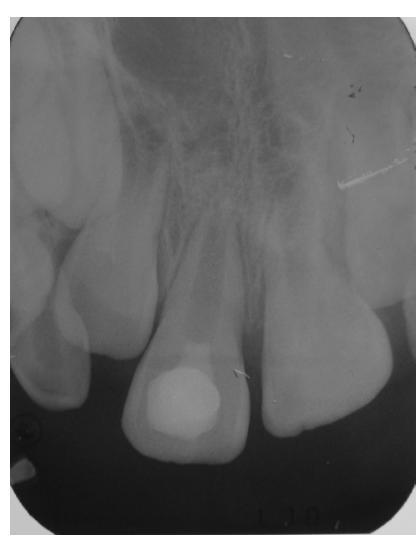

Figura 9. Control radiográfico. 


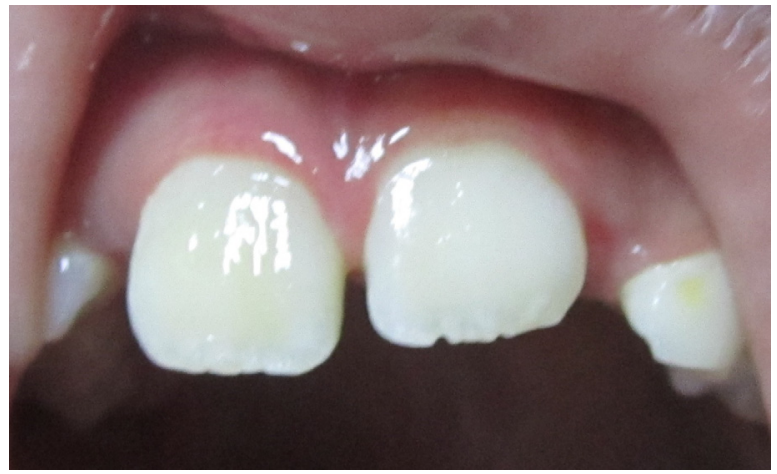

Figura 10. Sobre el MTA se colocó base de ionómero de vidrio y se reconstruyó con resina compuesta.

final, se realizaron controles clínicos y radiográficos para evaluar la ausencia de sintomatología y el desarrollo radicular; con lo que se puede concluir que el protocolo utilizado para este caso presentó resultados favorables. Observándose primero un engrosamiento de las paredes del canal radicular a los 04 meses de seguimiento, luego un aumento en su longitud radicular a los 07 meses y un posterior cierre apical a los 14 meses (Figura 11 - 14).

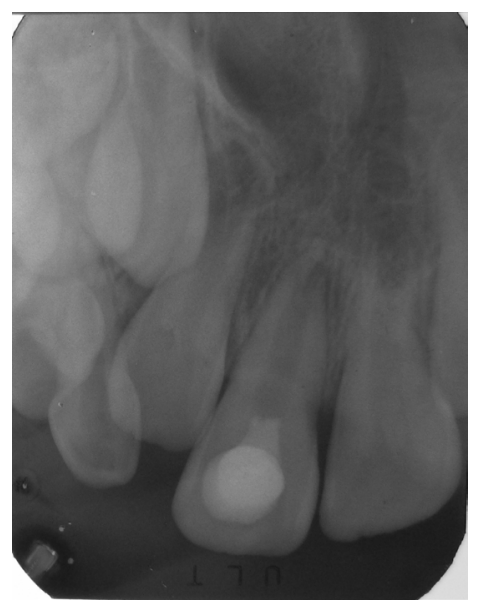

Figura 11. Pza. 11 mostró engrosamiento de las paredes del conducto radicular a los 4 meses post tratamiento.

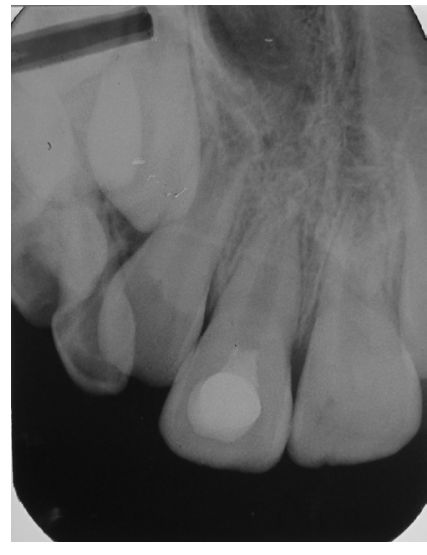

Figura 12. Pza. 11 muestra aumento de la longitud radicular a los 7 meses post tratamiento.

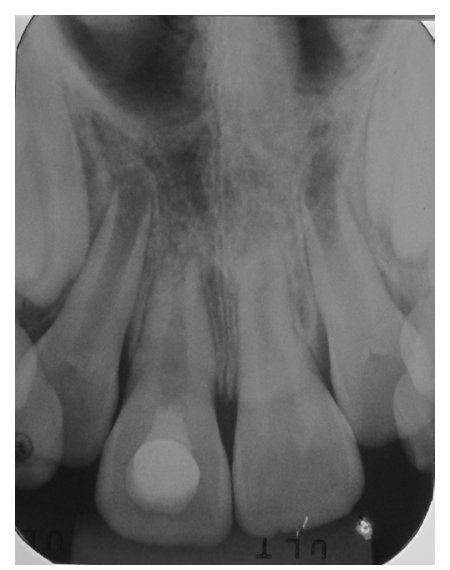

Figura 13. Pza. 11 se inicia formación del ápice radicular a los 10 meses post tratamiento.

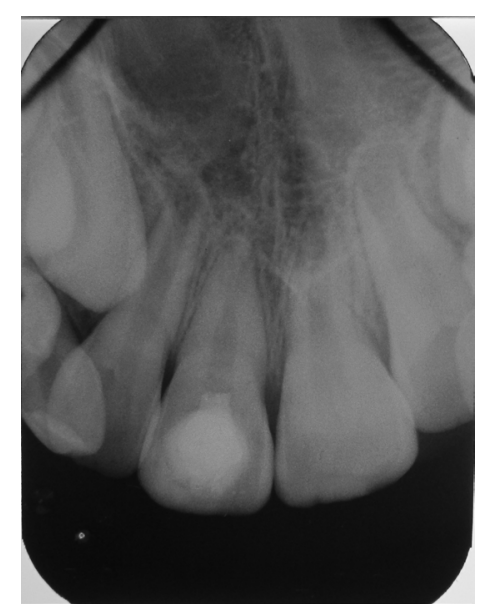

Figura 14. Pza. 11 muestra cierre apical formado a los 14 meses post tratamiento. 


\section{Discusión}

La apicoformación fue considerada por mucho tiempo la única opción para el tratamiento de dientes permanentes jóvenes no vitales, lamentablemente presenta varias desventajas como raíces cortas y propensas a la fractura; además de una proporción corona - raíz deficiente. Actualmente, estudios entre ellos Neha y cols. ${ }^{9}$ y Banchs y cols. ${ }^{14}$ proponen la revascularización como un tratamiento más conservador para estos dientes con resultados exitosos, así como lo demostró Ding y cols. ${ }^{10}$ en un estudio clínico de pacientes con diagnóstico de trauma dental y periodontitis apical.

El factor clave para el éxito del tratamiento de revascularización pulpar es la desinfección del sistema de conductos radiculares, así lo proponen Neha y cols. ${ }^{9}$ y Gómez. ${ }^{27}$ En el presente caso se realizó una mínima instrumentación, que sólo abarcó la entrada del conducto radicular, con lo que se busca facilitar la irrigación y el depósito de la medicación intra conducto; incluso algunos estudios realizados por Banchs y Trope $^{14}$ y entre otros proponen no realizar una instrumentación mecánica pues las paredes del conducto son delgadas. ${ }^{8,13}$

Namour y Theys ${ }^{6}$ y Fouad y cols. ${ }^{28}$ plantean el uso de irrigantes como el hipoclorito de sodio $(\mathrm{NaOCl})$ al 2,5\% asociado con el uso de EDTA al 17\%, pues éste último es quien permite que irrigantes y medicamentos puedan penetrar más profundamente a los túbulos dentinarios. En cambio, Wigler y cols. ${ }^{2}$ y Kharel y $\mathrm{Xu}^{5}$ recomiendan el uso de hipoclorito de sodio al $2.5 \%$ y clorhexidina al $2 \%$ para la desinfección de conductos, la cual fue usada también en el presente estudio, intercalando el lavado con cloruro de sodio para evitar la formación de precipitados. . $^{2,5,15,18,19}$

Como medicación intraconducto se utilizó la pasta $3 \mathrm{Mix}-\mathrm{MP}$, la cual tiene numerosos estudios que avalan su eficacia en la desinfección de conductos radiculares. ${ }^{12,13}$ Parasuraman y Muljibhai ${ }^{21}$ refieren que la utilización de propelinglicol como vehículo en la pasta 3Mix también favorece en la penetración de los medicamentos en los túbulos dentinarios. La principal desventaja en el uso de la pasta 3 Mix es la decoloración ocasionada por la minociclina y así lo sostienen algunos estudios. ${ }^{15}$

Por lo tanto, el presente estudio nos permite dar a conocer que la revascularización debería ser considerada como la primera opción de tratamiento en pacientes jóvenes.

\section{Conclusiones}

La revascularización es una opción de tratamiento conservador para dientes inmaduros, el cual permite un desarrollo radicular en sentido longitudinal y un engrosamiento de las paredes del conducto radicular con un posterior cierre apical.

\section{Referencias bibliográficas}

1. Nagy MM, Tawfik HE, Hashem AA, Abu-Seida AM. Regenerative potential of immature permanent teeth with necrotic pulps after different regenerative protocols. J Endod. 2014 Feb; 40(2):192-8.

2. Wigler R, Kaufman AY, Lin S, Steinbock N, Hazan-Molina H, Torneck CD. Revascularization: a treatment for permanent teeth with necrotic pulp and incomplete root development. J Endod. 2013 Mar; 39(3):31926. 
3. Sridharan S, Neelakantan I, Neelakantan P. Revascularization in endodontics. Int J Clin Dent. 2014; 7(2): $139-145$.

4. Shadmer E, Jenabi Dehkordi N. A Review on regenerative endodontics. JIDA. 2014; 26 (2): 116 - 126.

5. Kharel S, Xu Y. Revascularization: A promising alternative treatment for traumatic permanent immature necrotic teeth. JNDA. 2014; 14 (1): 1 - 7.

6. Namour M, Theys S. Pulp revascularization of immature permanent teeth: A review of the literature and a proposal of a new clinical protocol. ScientificWorldJournal. 2014; ID 737503: 1- 9.

7. Ahmed N, Neelakantan P. Antiseptics and antibiotics used in regenerative endodontics. IJPCR. 2013; 5 (4):141 - 144.

8. Dabbagh B, Alvaro E, Vu DD, Rizkallah J, Schwartz S. Clinical complications in the revascularization of immature necrotic permanent teeth. J Pediatr Dent. 2012; 34 (5): 414 - 417.

9. Neha K, Kansal R, Garg P, et al. Management of immature teeth by dentin - pulp regeneration: A recent approach. Med Oral Patol Oral Cir Bucal. 2011; 16 (7): 997 - 1004.

10. Ding RY, Cheung GS, Chen J, et al. Pulp revascularization of immature teeth with apical periodontitis: A clinical study. J Endod. 2009; 35 (5): 745 - 749.

11. Yassen GH, Vail MM, Chu TG, Platt JA. The effect of medicaments used in endodontic regeneration on root fracture and microhardness of radicular dentine. Int Endontic J. 2013; 46: 688 - 695.

12. Ordinola-Zapata R, Bramante C, Gagliardi P, et al. Antimicrobial activity of triantibiotic paste, $2 \%$ chlorhexidine gel, and calcium hydroxide on an intraoral - infected dentin biofilm model. J Endod. 2013; 39 (1): 115 - 118.

13. Windley W, Teixeira F, Levin L, Sigurdsson A, Trope M. Disinfection of immature teeth with a triple antibiotic paste. J Endod. 2005; 31(6): 439 - 443.

14. Banchs F, Trope M. Revascularization of immature permanent teeth with apical periodontitis: New treatment protocol? J Endod. 2004; 30 (4): 196- 200.

15. Kottoorr J, Velmurugan N. Revascularization for a necrotic immature permanent lateral incisor: A case report and literature review. Int J Paediatr Dent. 2013; 23: 310 - 316.

16. Deepak S, Nivedhitha MS. Clinical practice and guidelines and protocols for revascularization procedure - A review. Int J Pharm Sci Res. 2017; 9 (11): 2089 - 2092.

17. Shin SY, Albert JS, Mortman RE. One step pulp revascularization treatment of an immature permanent tooth with chronic apical abscess: a case report. Int Endod J. 2009;42(12):1118-1126.

18. Soares AJ, Lins FF, Nagata JN, Gomes BPFA, Zaia AA, Ferraz CCR, de Almeida JFA, Souza-Filho FJ. Pulp Revascularization after Root Canal Decontamination with Calcium Hydroxide and 2\% Chlorhexidine Gel. J Endod 2013; 39(3): 417-420.

19. Nagata JY, Rocha-Lima TF, Gomes BP, et al. Pulp revascularization for immature replanted teeth: a case report. Aust Dent J. 2015;60(3):416-420.

20. Vergara M, Díaz A, Alvear J. Eficacia de la pasta triantibiótica en conductos radiculares infectados con Enterococcus Faecalis. Revisión de literatura. RCV. 2013; 5 (1): 103 -108.

21. Parasuraman V, Muljibhai BS. 3 Mix - MP in Endodontics - An overview. JDMS. 2012; 3 (1): 36 - 45.

22. Quintana Q. Quispe M. Efectividad de una pasta tri-antibiótica en pieza decidua necrótica con absceso periapical y fístula. Odontol Sanmarquina 2012; 15(2): 31-34.

23. García - Godoy F, Murray P. Recommendations for using regenerative endodontic procedures in permanent immature traumatized teeth. Dent Traumatol. 2011; 1-9.

24. Chandran V, Chacko V, Sivadas G. Management of a nonvital young permanent tooth by pulp revascularization. J Clin Pediatr Dent. 2014; 7 (3): 213 - 216.

25. Velásquez V, Álvarez M. Tratamiento pulpar en la apexificación del diente inmaduro mediante agregado de trióxido mineral. Odontol Sanmarquina. 2009; 12 (1): 29 - 32.

26. Andreasen J, Bakland LK. Pulp regeneration after non - infected and infected necrosis, what type of Tissue do we want? A Review. Dent Traumatol. 2011; 28 (1): 13 - 18.

27. Gómez A. Regeneración endodóntica y revascularización pulpar ¿Una buena alternativa en endodoncia? Rev Sal Quintana Roo. 2012; 5 (19):19-22.

28. Fouad AF. The microbial challenge to pulp regeneration. Adv Dent Res. 2011; 23(3):285-289.

Recibido: $24 / 03 / 2020$

Aceptado: 06/08/2020

Correspondencia: Roxana Patricia López Ramos, correo: xxxx 